\title{
Partial and Full Inverse Compensation for Hysteresis in Smart Material Systems
}

\author{
Ralph C. Smith \\ Center for Research in Scientific Computation \\ North Carolina State University \\ Raleigh, NC 27695 \\ rsmith@eos.ncsu.edu
}

\author{
Chad Bouton \\ Battelle Memorial Institute \\ 505 King Avenue \\ Columbus, OH 43201 \\ bouton@battelle.org
}

\author{
Rick Zrostlik \\ Etrema Products, Inc. \\ 2500 North Loop Drive \\ Ames, IA 50010 \\ rick.zrostlik@etrema-usa.com
}

\begin{abstract}
Smart material transducers employing piezoceramic or magnetostrictive drive components typically exhibit constitutive nonlinearities and hysteresis at moderate to high drive levels. While feedback mechanisms or careful choice of operating regimes can often reduce these effects, spillover into high frequency dynamics and phase lags associated with the two phenomena will degrade the controller performance at high drive levels in the absence of additional compensation or nonlinear control design. In this paper, we discuss two techniques to compensate for hysteresis in high performance transducers. The first is based on a complete transducer model, and the resulting compensator accommodates both the constitutive nonlinearities and hysteresis inherent to the smart material components. The second technique employs a partial inverse compensator based on anhysteretic models for the material behavior. This accommodates the constitutive nonlinearities but does not incorporate the hysteresis; the latter phenomenon is then addressed through the inclusion of a feedback loop in the controller. The performance of the partial inverse compensator is illustrated in the context of a high force Terfenol-D transducer.
\end{abstract}

\section{Introduction}

Smart material transducers employing piezoceramic, electrostrictive or magnetostrictive cores provide the capability for both actuating and sensing in a wide range of high performance applications. Magnetostrictive transducers provide large forces with broader bandwidths than traditional magnetic shakers. Piezoceramic and electrostrictive patches have the capability for sensing and actuating while minimally affecting the passive dynamics of the underlying structure. Furthermore, they can be configured in a variety of geometries and are relatively inexpensive to manufacture. In combination with their dual sensing and actuating capabilities, these properties make them attractive in a number of control systems. In their biased state, all of these materials exhibit an approximately linear behavior at low drive levels. At moderate to high drive levels, however, all exhibit constitutive nonlinearities including saturation effects. Furthermore, piezoceramics and magnetostrictives exhibit significant hysteresis while relaxor ferroelectrics are hysteretic when employed in their ferroelectric phase. These constitutive nonlinearities and hysteresis must be accommodated to achieve the full capabilities of the materials in high performance control design.

A common strategy is to limit input levels to ranges in which the material behavior is approximately linear. This permits the use of linear models and control designs. While feasible in certain applications, this significantly limits the capability of the transducers. For piezoceramic materials, current control in lieu of voltage control can be used to maintain an approximately linear response [3]. However, these techniques do not appear directly applicable to magnetostrictive transducers. For certain applications, feedback loops can be used to reduce the effects of hysteresis. For high accuracy or micropositioning applications, however, further compensation is typically required. This can be achieved either through the development of inverse compensators incorporated in linear control designs or nonlinear controllers. In this paper, we focus on the development of full or partial inverse compensators and refer the reader to [4] for an example of nonlinear optimal control design in the context of a Terfenol-D magnetostrictive transducer. 
The concepts underlying full and partial inverse compensation are illustrated in Figure 1. In both cases, we assume that the smart material transducer provides hysteretic inputs to the system. For full inverse compensation, a complete inverse $N^{-1}$ is constructed to accommodate the actuator hysteresis $N$ so that the control input $u$ to the plant is equal (or approximately equal) to the desired input $u_{d}$ specified by the control law. This inverse filter is constructed using the complete model used to characterize the hysteretic transducer. The partial inverse compensator considered here accommodates the constitutive nonlinearities through the anhysteretic curve. The uncompensated hysteresis then causes a phase delay between $u$ and $u_{d}$ which is accommodated through a feedback loop. This places additional impetus on the control design but significantly reduces the overhead required for implementation.

The domain wall model used to characterize the hysteresis is summarized in Section 2. This model is advantageous in that it is equally applicable to ferromagnetic and ferroelectric materials and it can be inverted to provide a full compensator for linear control design. Furthermore, the anhysteretic component provides the filter employed in partial compensator design. The development of partial and full inverse compensators is summarized in Section 3. The performance of the partial inverse compensator is illustrated in Section 4 for a high speed, high accuracy milling application.

\section{Hysteresis Model}

The model described here is based on the tenet that hysteresis in ferromagnetic and ferroelectric materials is due to the impeded movement of domain walls which are pinned at inclusions or defects in the material. For brevity, we focus on on the modeling of hysteresis in

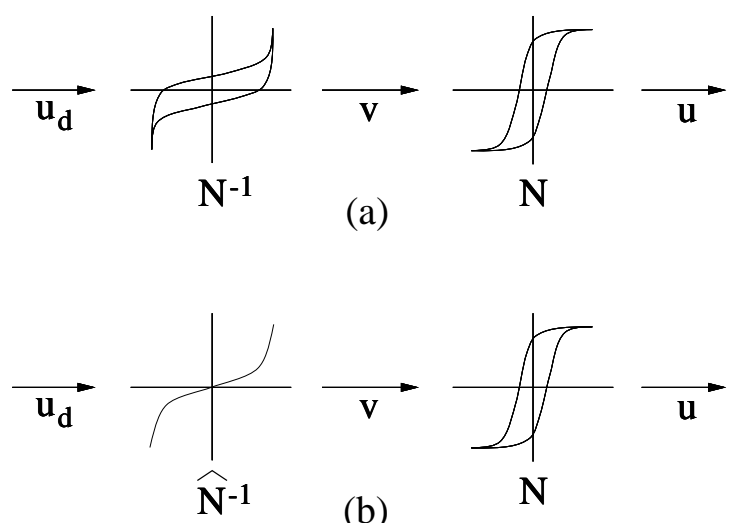

Figure 1. (a) Full inverse compensator employed as a filter for linear control design; (b) Partial inverse compensator based on the anhysteretic curve. ferromagnetic materials throughout the remaining discussion. The reader is referred to [6] for an analogous development for general ferroelectric materials and [7] for details regarding the use of the model to characterize hysteresis in piezoceramic materials.

The model development is considered in two steps. In the first, the anhysteretic magnetization $M_{a n}$ is quantified using two techniques; the first is based on Boltzmann statistics while a polynomial fit comprises the second. The second step in the model development is the quantification of the irreversible magnetization $M_{i r r}$ and reversible magnetization $M_{\text {rev }}$ which result when domain walls respectively translate and bend. The sum of the two components yields the total magnetization.

\subsection{Anhysteretic Models}

The anhysteretic magnetization at a point in the material is dependent on the effective magnetic field, the saturation magnetization and the thermal energy of the sample. Under the assumption of constant stress $\sigma_{0}$, the effective field can be expressed as $H_{e}=H+\alpha M$ where $H$ denotes the external field input to the transducer, $M$ denotes the magnetization and the parameter $\alpha$ quantifies the effects of interdomain coupling and magnetoelastic domain interactions. The balance of thermal and magnetostatic energy using Boltzmann principles respectively yields the Langevin and Ising spin models

$$
\begin{aligned}
& M_{a n}=M_{s} \mathcal{L}\left(H_{e} / a\right) \\
& M_{a n}=M_{s} \tanh \left(H_{e} / a\right)
\end{aligned}
$$

for the anhysteretic magnetization. Here the Langevin function is defined by $\mathcal{L}(z)=\operatorname{coth}(z)-1 / z$. The parameters $\alpha$ and $a$ are estimated for a given transducer through a least squares fit to experimental data.

A second technique for modeling the anhysteretic magnetization is through the use of piecewise polynomials or general power law representations fit to data measured in specific applications. For example, a piecewise fit employing three components has the form

$$
M_{a n}=\left\{\begin{array}{l}
f_{1}(H), H_{\min } \leq H \leq H_{1} \\
f_{2}(H), H_{1}<H \leq H_{2} \\
f_{3}(H), H_{2}<H \leq H_{\max }
\end{array}\right.
$$

where $f_{1}(H), f_{2}(H), f_{3}(H)$ are piecewise functions and $H_{\min }, H_{\max }$ are the minimum and maximum field inputs. The determination of specific functions to attain an appropriate inverse is illustrated in Section 4 .

\subsection{Full Hysteresis Model}

As detailed in [1], the irreversible magnetization is quantified by determining the energy required to reorient dipoles in the presence of an applied field. This 
yields the differential equation

$$
\frac{d M_{i r r}}{d H}=\widetilde{\delta} \frac{M_{a n}-M_{i r r}}{k \delta-\alpha\left(M_{a n}-M_{i r r}\right)}
$$

where the parameter $k$ provides an average measure of the energy required to re-orient domains and hence translate domain walls. The parameter $\delta$ is defined to have the value +1 when $d H>0$ and -1 when $d H<0$ to guarantee that pinning always opposes changes in magnetization. In applications, $\delta$ can be directly determined from the magnetic field data. Finally, the parameter $\widetilde{\delta}$ is defined to be 0 if $\left\{d H>0\right.$ and $\left.M>M_{a n}\right\}$ or $\{d H<$ 0 and $\left.M<M_{a n}\right\}$ and is 1 otherwise to incorporate the physical observation that following field reversal at the loop tip, the changes in magnetization are reversible until the anhysteretic value is reached [2].

The second component of the magnetization is the reversible magnetization which reflects the degree to which domain walls bend before attaining the energy necessary for translation. As derived in [1], the inclusion of this component yields the total magnetization

$$
M=(1-c) M_{i r r}+c M_{a n}
$$

where $M_{i r r}$ is defined by (3) and the anhysteretic magnetization $M_{a n}$ is given by (1) or (2).

To provide a relation which facilitates inversion, it is advantageous to express the output magnetization as a function of the input field. As detailed in [5], the magnetization at a given field level can be specified as the solution to the differential equation

$$
\frac{d M}{d H}=\mathcal{F}(H, M)
$$

where $\mathcal{F}$ is given by

$$
\begin{aligned}
\mathcal{F}(H, M)= & \frac{1}{1+c M_{s} \alpha \frac{\partial \mathcal{L}}{\partial H}\left(\frac{H+\alpha M}{a}\right)} \\
\bullet & \left\{\frac{\tilde{\delta}\left[M_{s} \mathcal{L}\left(\frac{H+\alpha M}{a}\right)-M\right]}{k \delta-\tilde{\alpha}\left[M_{s} \mathcal{L}\left(\frac{H+\alpha M}{a}\right)-M\right]}\right. \\
& \left.-c M_{s} \frac{\partial \mathcal{L}}{\partial H}\left(\frac{H+\alpha M}{a}\right)\right\} .
\end{aligned}
$$

\subsection{Full Model Inverse}

The hysteresis model in the form (5) is amenable to inversion through consideration of the complementary differential equation

$$
\frac{d M^{-1}}{d H}=\frac{1}{\mathcal{F}\left(M^{-1}, H\right)}
$$

with $\mathcal{F}$ defined by $(6)$. This provides the exact inverse $N^{-1}$ if the parameters $M_{s}, \alpha, a, c$ and $k$ are known exactly or an approximate inverse $\widetilde{N}^{-1}$ if the parameters are unknown or slowly changing due to changing operating conditions. Details regarding the design of control methods which employ an approximate inverse can be found in [9].

\section{Full and Partial Inverse Compensators}

The previously described anhysteretic and hysteresis models provide the capability for constructing both full and partial inverse compensators for linear control design. To construct a full inverse compensator, the differential equation (7) is used to construct a filter $N^{-1}$ which is employed in the manner illustrated in Figure 1a. This provides a method for linearizing the system when the degree of hysteresis is large. Details regarding the performance of the full inverse compensator for a Terfenol-D magnetostrictive transducer are provided in [8]. We note that for the full inverse to be successful, it is important to have accurate initial conditions which is difficult to attain experimentally. This is one motivation for considering a partial compensator.

A partial inverse compensator can be constructed using the Ising spin model (1) or piecewise power representation (2). The resulting inverse incorporates the nonlinear behavior, including saturation effects, but neglects the phase delays due to hysteresis (see Figure 1b). For systems with moderate hysteresis, these latter effects can be treated as a disturbance and targeted with a feedback loop. A simple control design utilizing the partial inverse compensator and feedback loop is depicted in Figure 2. We note that this design was used to establish the potential capabilities of the partial compensator and is not optimal. We are currently investigating the placement of the partial compensator inside the feedback loop along with adaptive temperature compensation. Examples illustrating the performance of the partial inverse compensator are provided in the next section.

\section{Control Implementation}

We illustrate in this section the implementation of the control strategies depicted in Figure 2 in the context of a high speed Terfenol-D driven milling application shown in Figure 3. This represents a prototypical application of the type developed at Etrema Products, Inc. The milling specifications include a $3000 \mathrm{rpm}$ rotation rate and cutting tolerances of approximately \pm 1 micron.

The inverse to the anhysteretic curve for the application was specified to be

$I_{a}\left(x_{c}\right)=\left\{\begin{array}{l}0.55 x_{c}^{0.51}, 0 \leq x_{c}<190 \\ 0.0106 x_{c}+5.966,190 \leq x_{c}<450 \\ 0.45 \times 10^{-4}\left(x_{c}-350\right)^{2.2}+9.6, x_{c} \geq 450\end{array}\right.$

to accommodate the slight degree of asymmetry exhibited by the data (see Figure 4). This represents the inverse of the general anhysteretic representation in (2). 


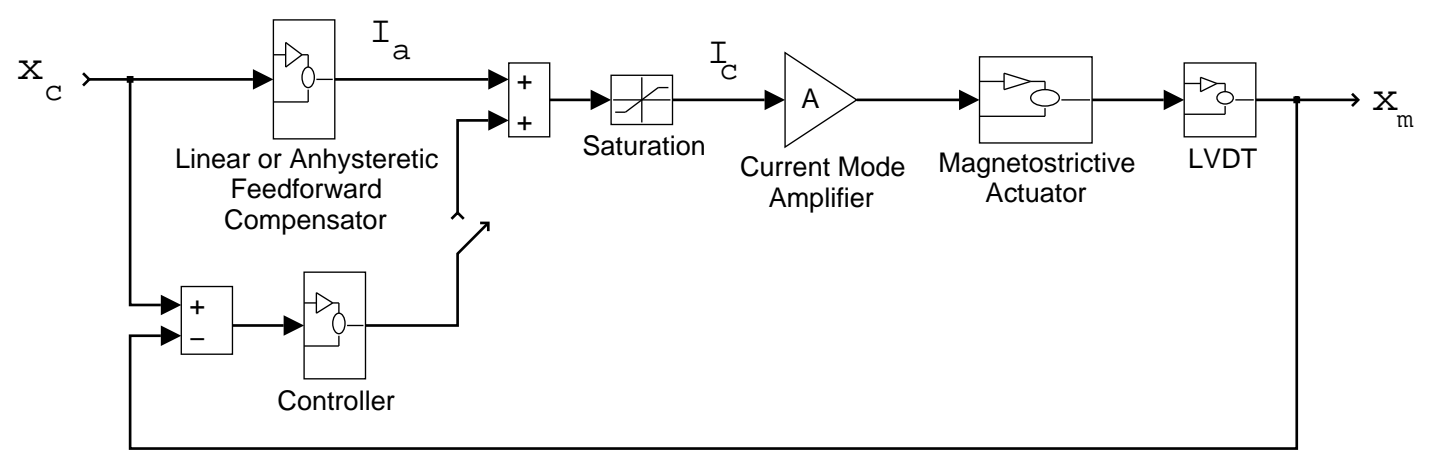

Figure 2. Control design utilizing the partial inverse compensator in a feedforward loop. A feedback loop targets the disturbance due to hysteresis.

The performance of three control designs is summarized in Figure 4. The first consists of an open loop control law specifying the commanded position with no compensation for nonlinearities or hysteresis. As illustrated by the phase plot comparing the commanded and measured cutting head positions, there are significant saturation effects and hysteresis which then produces errors in excess of 70 microns when the measured position is considered as a function of time. The second design utilizes the partial inverse compensator, as illustrated in Figure 2, but without the feedback loop. From the phase plot in Figure $4 \mathrm{~b}$ it is observed that this eliminates most of the nonlinearity due to saturation but neglects the inherent hysteresis. Hence there is a slight phase delay in the time history of the measured cutting head position. Figure $4 \mathrm{c}$ illustrates the performance of the partial inverse compensator when the feedback loop is included. While operating under a slightly different command signal, it can be observed that the feedback adequately attenuates the disturbances due to hysteresis for the operating regime. This design produced a cutting accuracy of \pm 15 microns which has subsequently been reduced to \pm 2 microns using a narrowband control.

In summary, the use of a partial inverse compensator, based on the anhysteretic magnetization or polarization, provides a viable design tool for certain hysteretic smart material systems when combined with a feedback loop

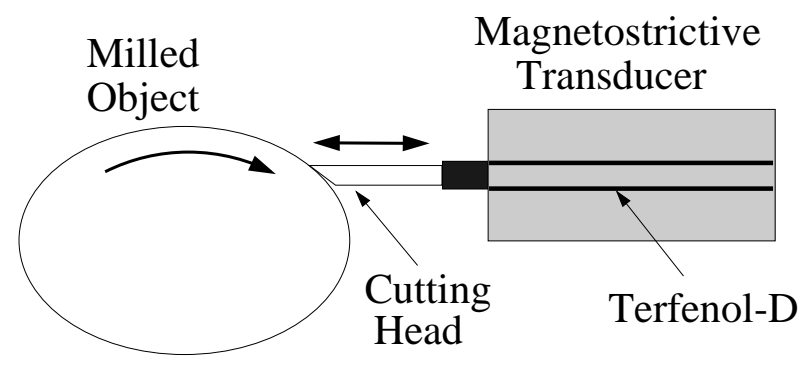

Figure 3. High speed milling application. which targets the hysteresis. The advantage of this technique is the efficiency with which it can be implemented and the observation that it appears less sensitive to accurate initial conditions than the full inverse compensator. For systems which exhibit significant hysteresis, however, the full inverse compensator may be required to attain the design specifications including accuracy and minimal phase delays.

\section{Acknowledgements}

The research of R.C.S. was supported in part by the Air Force Office of Scientific Research under the grant AFOSR-F49620-98-1-0180.

\section{References}

[1] D.C. Jiles and D.L. Atherton, "Theory of ferromagnetic hysteresis," Journal of Magnetism and Magnetic Materials, 61, pp. 48-60, 1986.

[2] D.C. Jiles, J.B. Thoelke and M.K. Devine, "Numerical determination of hysteresis parameters for the modeling of magnetic properties using the theory of ferromagnetic hysteresis," IEEE Transactions on Magnetics, 28(1), pp. 27-35, 1992.

[3] J.A. Main and E. Garcia. "Piezoelectric stack actuators and control system design: strategies and pitfalls." Journal of Guidance, Control, and Dynamics. 20(3), pp. 479-485, 1997.

[4] R.C. Smith, "A nonlinear optimal control method for magnetostrictive actuators," Journal of Intelligent Material Systems and Structures, 9(6), pp. 468-486, 1998.

[5] R.C. Smith, "Inverse compensation for hysteresis in magnetostrictive transducers," CRSC Technical Report CRSC-TR98-36; Mathematical and Computer Modeling, to appear. 

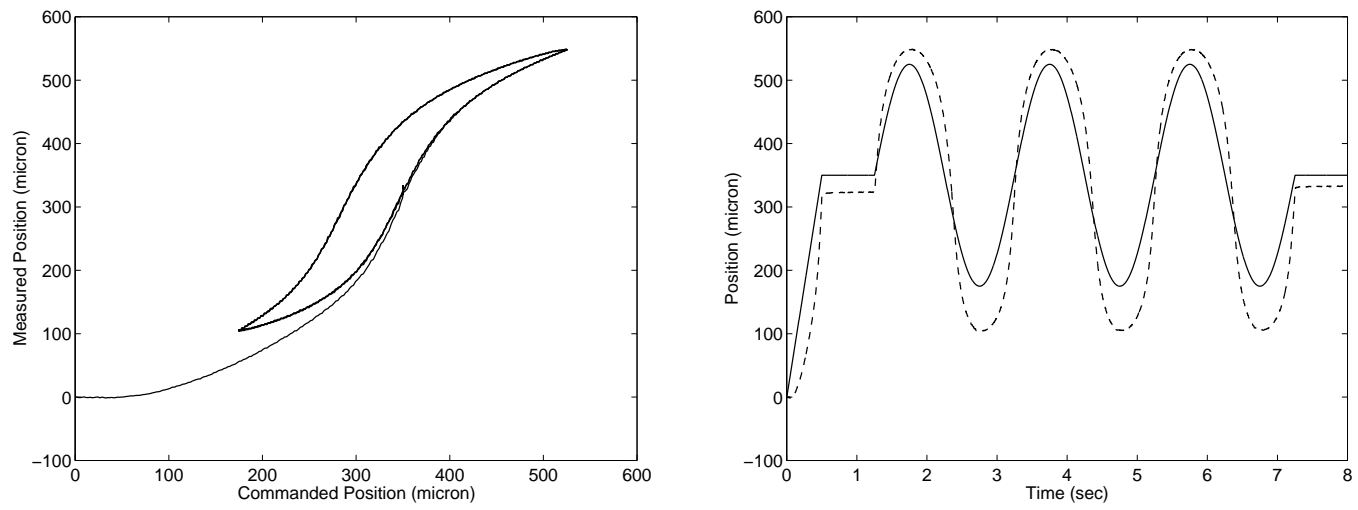

(a) Open Loop Control with no Compensation or Feedback
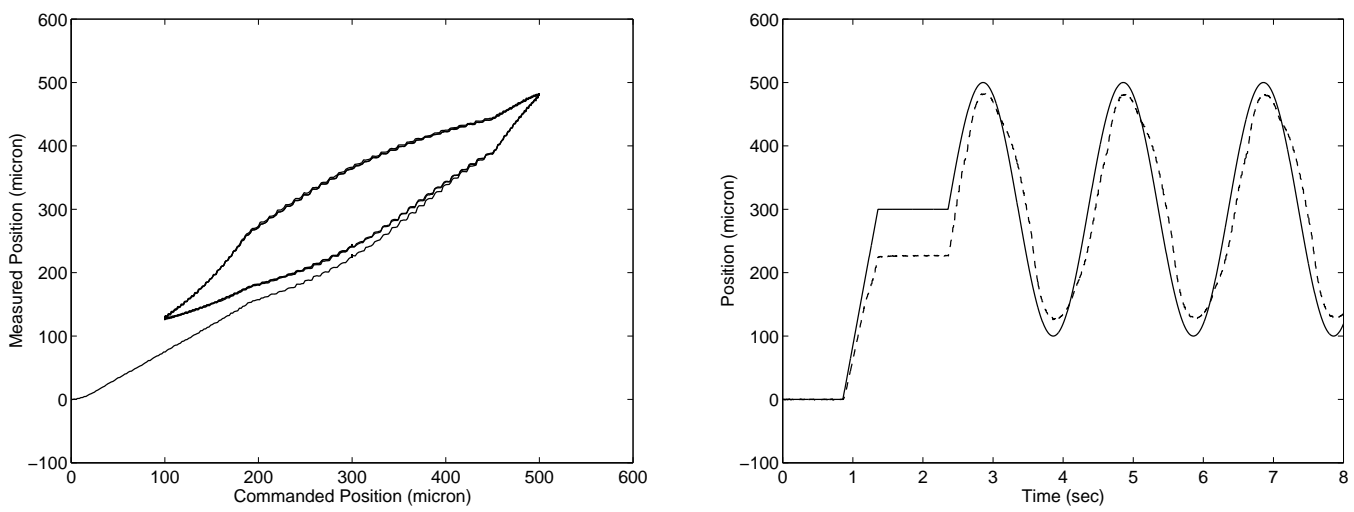

(b) Partial Inverse Compensation but no Feedback
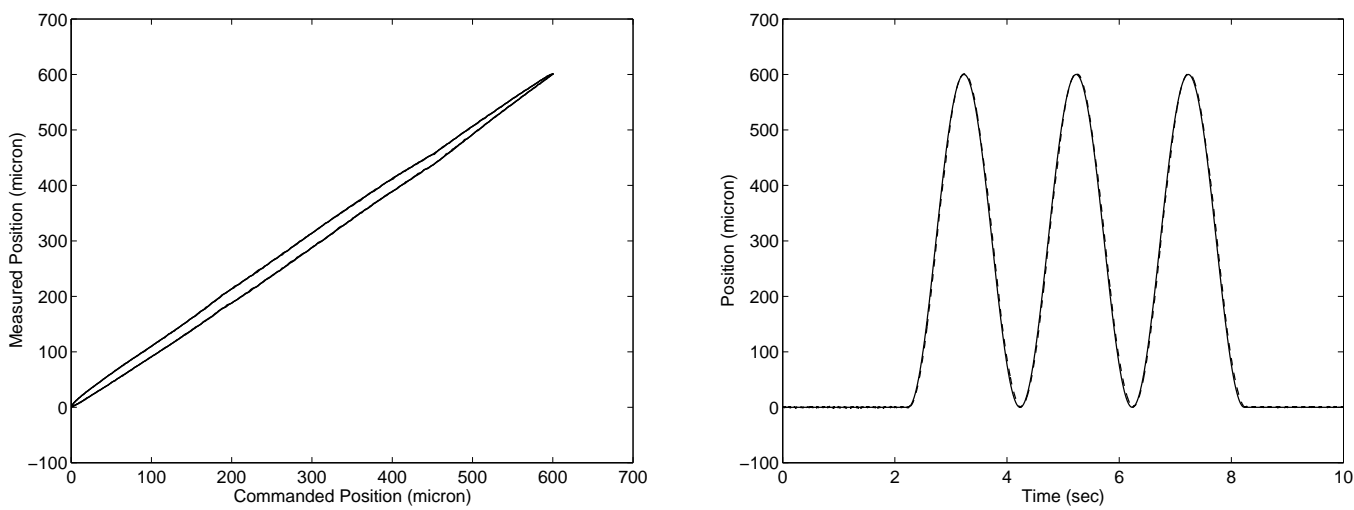

(c) Partial Inverse Compensation and Feedback

Figure 4. Left: Measured versus commanded cutting head position. Right: Commanded position (- - ) and measured position (-- ) of the cutting head as a function of time.

[6] R.C. Smith and C.L. Hom, "A domain wall theory for ferroelectric hysteresis," CRSC Technical Report CRSC-TR99-1; Journal of Intelligent Material Systems and Structures, to appear.

[7] R.C. Smith and Z. Ounaies, "A domain wall model for hysteresis in piezoelectric materials," CRSC Technical Report CRSC-TR99-33; Journal of Intelligent Material Systems and Structures, submitted.
[8] R.C. Smith and R. Zrostlik, "Inverse Compensation for Ferromagnetic Hysteresis," CRSC Technical Report CRSC-TR99-28; Proc. 38th IEEE Conf. Dec. and Control, Phoenix, AZ, 1999.

[9] G. Tao and P.V. Kokotović, Adaptive Control of Systems with Actuator and Sensor Nonlinearities, John Wiley and Sons, New York, 1996. 